\title{
Possible Improvements of Acupuncture for Knee-Pain Treatment Outcomes Through Local Point Palpation
}

\author{
Vladislav Korostyshevskiy, LAc, MS, MEd
}

\begin{abstract}
Background: Much of the world's population suffers from knee pain. Treatment options are too extreme (surgery), have side-effects (drugs), or take too long (physical therapy). Research has shown that acupuncture can provide modest relief of knee pain. This article presents ways to improve the effectiveness of acupuncture for treating knee pain. Using 3 composite "cases," the author offers specific modifications to acupuncture treatments - particularly palpatory techniques - that produce more-successful relief of patients' knee pain.

Cases: Analyzing 3 composites of multiple similar typical cases, the author compares what made the treatments more successful than would be statistically anticipated. Composite case 1: Women, older than, 50 had knee osteoarthritis (OA), periodic pain, more at night and during weather changes. Composite case 2: Men and women, in their 40s and early 50s, had mild knee arthritis, and were given injections to relieve pain that was mild-to-moderate and worse at night. Composite case 3: Men between their late 20s and early 40s, experienced knee pain 1-4 times per week, with some patients reporting knee buckling after some activities.

Results: Palpation of these patients' knees revealed the causes of their knee pain-spasms, adhesions, and/or fibrosis-and the patients were treated with individually selected points based on results of the Four Examinations. Most obtained relief lasting from 4 months to 2 years.

Conclusions: Mainstream acupuncture treatments rarely involve using the Four Examinations of Traditional Chinese Medicine. Instead, these treatments involve common point selections for addressing knee pain or moreadvanced approaches, such as Five Elements or Zang-Fu pattern diagnosis, often disregarding such basic but essential diagnostic techniques as visual inspection and particularly palpation. According to the Four Examinations, OA is not often the cause of knee pain; instead, adhesions and fibrosis of the soft tissues around the knee joint cause nerve entrapments, and knee pain is referred from those sources. While muscle fibrosis is well-known to cause pain, it is rarely addressed in detail in acupuncture literature. If palpation of the soft tissues around the knee joint evokes a patient's pain-thus, locating the nerve entrapment in each specific case precisely-this allows the clinician to insert acupuncture needles into the soft-tissue fibrosis. This needling could improve the clinical outcomes of acupuncture treatment of knee pain significantly, providing shorter- as well as longer-term relief.
\end{abstract}

Keywords: knee pain, acupuncture, palpation, knee arthritis, Traditional Chinese Medicine (TCM)

Private practice, Brooklyn, NY, USA. 


\section{INTRODUCTION}

$\mathbf{K}$ NEE PAIN IS THE MOST COMMON CAUSE of pain among adults 50 years old and older and reduces quality of life significantly for people who have this pain. ${ }^{1-5}$ Pharmacologic treatment offers limited relief from pain but can also cause various adverse effects. Surgeries, such as knee replacement, are extreme and expensive alternatives resorted to when the knee joint is damaged. ${ }^{1}$ Physical therapy comprises a variety of modalities that can reduce knee pain ${ }^{2}$ and improve coping skills in people who have osteoarthritis (OA) of the knee. ${ }^{3}$

Research has also shown that acupuncture can be modestly effective for treating knee pain. ${ }^{4,5}$ The mainstream approach to acupuncture treatments used in modern clinical settings - the same approach reflected in research studiesis rarely tailored to individual patients' knee problems. This can be noted by examining the local acupuncture points selected in many research studies: These points are typically the ones recommended in the acupuncture textbooks that are also used as standards in acupuncture-training programs. If researchers would, instead, rely on the Four Examinations, however, then point selections as well as locations would have to differ almost always for each case.

Acupuncture practitioners also tend to either use popular local and distal points that are widely accepted as proper treatments for knee pain, or some practitioners tend to use more-advanced methods, such as Zang-Fu differential diagnosis or Five Elements treatment, skipping a large range of diagnostic possibilities that the Four Examinations would make possible. This might be the reason for acupuncture's modest clinical success for treating knee pain.

This article illustrates the variations in diagnoses and treatments of knee pain that were modified according to observation, inquiry, and palpation. The current author tested this approach during his 20 years of clinical experience of treating primarily patients with musculoskeletal issues. This article also offers an opinion, rather than presenting a formal study. Instead, there are descriptions of 3 typical "cases" from the author's practice in which the patients' knee pain was resolved completely. Each case is a composite of at least 12 similar cases from which patterns can be derived.

\section{Local Acupuncture Points for Knee Pain Used in Research Studies}

There are significant variations in the way knee pain is examined with acupuncture in various acupuncture research studies. For example, in 1 study conducted on athletes, the researchers stated that the acupuncture treatment protocol that they used was "based on pressure points of systemic acupuncture with evidence supporting a decrease in pain symptomatology in the knee region." 6 The selected points were: (1) ST 35 (Dubi); (2) EX-LE 4 (Neixiyan); and (3) the cranial point contralateral to the affected limb. Curiously, all participants in this research reported reductions in knee pain after treatment, and the affected limbs had improvements in average strength by $34 \%$ and $25 \%$, respectively, compared to the contralateral limbs. ${ }^{6}$ This is a high success rate, which might have been due to the researchers' monitoring their point selections via palpation. Palpation was used, which is an integral component of the Four Examinations, in a specific way - the researchers confirmed that the points that they chose were sensitive on each subject before beginning the treatments. Palpation, however, can be used more extensively. This can be performed-not only on the classical points but also on the adjacent areas of the leg-to examine the tissues that surround the knee for any possible contribution to the presented symptoms (i.e., light-to-moderate pressure applied to various points around the knee might relieve or worsen the intensity of the patient's knee pain).

In other studies, the participating acupuncturists decided what points they would use, and their point selections ranged from the classical traditional Chinese methods ${ }^{7}$ to blends of the traditional Chinese and modern medical approaches. ${ }^{8}$ No matter what approach was used, however, the outcomes appeared to be consistent among various studies: there were short-term benefits at the end of treatment, but these benefits seemed to disappear over time. ${ }^{7}$ This consistency in findings is hardly surprising, considering that the local point selections in many studies are similar; local points are typically selected from the acupuncture points that most commonly used textbooks recommend for treating knee pain. From the author's experience, however, acupuncture can be more effective for treating this pain. The main problem causing the modest success rates is not in the research designs per se but exists in the way acupuncture is practiced today.

It would make perfect sense for acupuncture practitioners to rely on an official diagnosis of OA of the knee made by an orthopedist instead of doing their own visual inspectionslistening/smelling, questioning, and palpation-which comprise the Four Examinations. While there is little doubt that at least some acupuncture practitioners use the Four Examinations regularly, as some of the references used in this article illustrate, it appears that specific techniques of the Four Examinations (with special emphasis on palpation in this article), when used by acupuncture practitioners, can play a significant role in clinical success rates. Generally speaking, the goal is to find sensitive points-which is hardly new-but the ways sensitivity varies and the ways the tissues feel under the fingertips can play important roles in the treatment chosen and its outcome.

\section{COMPOSITE CASES}

\section{Composite Case 1}

This was the most common category, which occurred primarily in women over 50 years old. The patients often 
came in with the diagnosis of OA (established by an orthopedist) of either 1 knee or both knees. These patients reported experiencing periodic pain, more so at night-time and when the weather changed. The first instinct a clinician might have had was to use such common points for addressing knee pain, such as ST 35 (Dubi) or EX-LE 4 (Neixiyan), but, visually, the knees were not red or swollen or deformed in $\sim 80 \%$ of these cases.

While these criteria might deviate from the consensus of what reliable symptoms and signs of OA of the knee are, ${ }^{9}$ these criteria indicate obvious and rather advanced OA of the knee. To increase acupuncture's success rates, all other symptoms and signs can be evaluated using the Four Examinations, which help identify the precise location of peripheral nerve entrapment, as well as discovering if there is one.

Light palpation revealed no difference in temperatures between the knee and the surrounding tissues, but this palpation revealed abnormal variations in soft-tissue textures. The surrounding knee muscles and adjacent soft tissues appeared to have fibrosis and adhesions, more so in the medial aspect of the legs, distally as well as proximally to the knees. More importantly, however, was that light-tomoderate pressure palpation applied in pinch-like fashion ${ }^{10}$ to certain areas in the tissues caused sharp, intense pain in these patients.

It is known that "[s]keletal muscle fibrosis is a major pathological hallmark of chronic myopathies," 10 but the current author never saw this issue addressed by acupuncture treatment directly, although the Traditional Chinese Medicine (TCM) concept of Ah Shi points allows it. ${ }^{11}$ To monitor treatment progress, it is preferred that the pressure from the palpation reproduces the same pain that the patients complain about. However, in this case category, the patients often were not sure if the pain was the same. Nonetheless, if a light-pressure pinch $^{12}$ produces sharp pain, it indicates inflammation of a local nerve. Palpation with the same pressure along the nerve pathway proximally and distally from the painful point showed that pain decreased and then disappeared in both directions, leading to the acupuncturist to believe that adhesions and fibrosis were the causes of the nerve inflammations. Further careful lightpressure palpation vertically to the surface of the skin also revealed that certain small areas in the medial aspect of the knee had a drumlike quality.

One-inch, 38-gauge needles were inserted in those points. The points were already sensitive, and the current author did not stimulate the needles to ensure arrival of the De Qi sensation. ${ }^{13}$ The needles were then left inserted for 20 minutes and gently stimulated every 5 minutes.

During the second session, in the majority of cases, palpation revealed that the drumlike areas had become smaller, and the patients reported that their knee pain had become less intense. The same treatment strategy was repeated. On the third visit, the patients usually reported that their knee pain had decreased in intensity and frequency, and finding the local points that had the same drumlike quality was more challenging. On the fourth visit, the patients typically reported that they felt only infrequent mild pain, and the current author was able to find only very small points that still had that drumlike quality. While the number of sessions usually fluctuated between 3 and 7, often their number appeared to depend on the acupuncturist's ability to find the right points more than any other factors. The patients typically reported experiencing periodic pain in their knees that was much milder. The majority of patients in this category, however, reported that their pain returned between 4 and 8 months after the treatments were finished.

\section{Composite Case 2}

This category involved patients in their 40s and early 50s, equally distributed among men and women, who presented with the chief complaint of knee pain. Patients who had seen orthopedists were diagnosed with mild knee arthritis and told that it was too early to think about knee replacement; instead, pain-relieving injections were recommended. Patients who received the injections reported that their pain went away after the injections but this relief lasted for only a few days. Most patients described their pain as resembling a mild-to-moderate toothache in quality and intensity. The pain usually was worse at rest and at night-time and was not as intense during most activities.

Palpation of the anterior, medial, and lateral aspects of the knee revealed nothing abnormal in the majority of these cases. Considering the high frequency and intensity of the pain, there had to be something abnormal that should be possible to identify via palpation. The current author then pressed on the posterior aspect of the knee in each patient; this evoked the patient's pain. Further palpation of each patient's knee while he or she was in a prone position on the treatment table revealed soft-tissue adhesions in the area of the popliteal fossa, and soft-tissue adhesions and fibrosis in the leg immediately distal and proximal to the knee.

None of the patients reported having traumata to their legs but they all had jobs that involved siting long for long hours, such as computer programming or accounting. Their legs might have been pressed against the edge of their chairs at work, which might have presumably been the cause of the adhesions and fibrosis.

Most of the patients reported that their pain had decreased after the second treatment and had completely resolved after the seventh session. While the point selections were different during each session, the criteria for selection remained the same for each patient: nodules of various degrees of firmness and light-to-moderate pinch-like pressure on the knee that reproduced the patient's pain. Between 8 and 2038 -gauge $1<$ inch mark> needles were used in every session, depending on the number of active points that were identified. The patients reported that their pain did not recur for 2 years following their last treatment sessions. 


\section{Composite Case 3}

The patients in this category were between late their 20s and early 40s, and were primarily males who reported feeling pain in their knees between 1 and 4 times per week. This condition lasted for several months or even a few years, and some patients reported that their affected knees had buckled after walking, running, or riding bicycles for a few miles. Orthopedists typically found nothing wrong with the patients' affected knees and told some patients that their problems originated in their lower backs. While magnetic resonance imaging reports often described disc herniation, $\sim 12$ physical therapy sessions made little or no difference in the patients' symptoms.

Palpation of the patients' affected knees and legs usually revealed only muscle spasms in the affected areas-including ST 34 (Liangqiu) and EX-LE 2 (Heding), - but, more importantly, moderate-pressure palpation on the firmest points in the spasm reproduced the patients' knee pain. Considering that the current author had seen patients with identical complaints and the accompanying muscle spasm in the area of ST 34 (Liangqiu), that spasm was most likely the culprit causing the knee pain and buckling.

These patients reported feeling no more pain and knee buckling after between 3 and 7 sessions of inserting $1<$ inch mark>, 38-gauge acupuncture needles in the tightest points in the area of ST 34 (Liangqiu). The pain typically did not return from six months to 2 years after the treatments were finished.

\section{RESULTS}

Composite case 1 patients had between 3 and 7 sessions. Each session resulted in the patients experiencing less knee pain. After the treatments were finished, the patients had relief of their pain for 4-8 months before their pain recurred.

Composite case 2 patients had 7 sessions with different point selections depending on the nodules found and pain evoked by light-to-moderate pinch pressure. After their final sessions, the patients reported that their pain resolved completely and did not recur for 2 years afterward.

Composite case 3 patients had 7 sessions with points selected in the tightest areas of their spasms. Between sessions 3 and 7, these patients reported having no more pain and knee buckling. Their pain did not recur from 6 months to 2 years.

\section{DISCUSSION}

While TCM includes the Four Examinations in its arsenal of diagnostic tools, palpation appears to be particularly important and intricate (although, visual inspection and questioning the patient need to go together with palpation).
Considering that acupuncture points are small, intuition might suggest pressing on the points vertically to the skin surface. While, in certain circumstances, this kind of palpation can be helpful or even essential, initially, it provides only limited information. The problem is that, as human beings move, many of these movements involve stretching of some tissues and compression of others.

By compressing a suspicious area in a pinch-like way, the practitioner is more likely to reproduce the pain that occurs naturally, than when pressing vertically down on points. Paying careful attention to the thickening, tightening, and stiffening of the soft tissues under the fingertips, practitioners will be able to identify the exact locations of nerve entrapments. Vertical pressure becomes important during the next stage of palpation, when practitioners are palpating for the exact location of the needling site and the best insertion angle. When active points ${ }^{14}$ are located in the medial aspect of the knee-where the muscle tone is often low (ancient TCM practitioners explained that the meridians in that area are Yin and that they can become Deficient)careful light-pressure palpation in which the fingertips slide across the skin surface (the current author prefers to do this kind of palpation in a spiral) still reveals a drumlike quality over the active points.

Needle insertion in such active points requires minimal stimulation to cause the arrival of De $\mathrm{Qi},{ }^{13}$ because an Excess of Qi is already in that location (due to the local inflammation). Thus, additional point stimulation will cause unnecessaryand often intense-pain. At the same time, if the inserted needle slightly misses the point, then additional stronger stimulation can improve the treatment outcome by bringing more Qi to the actual point. Yet, the points along the Stomach (ST) and Gallbladder (GB) meridians (Yang meridians) typically do not have that drumlike quality; they usually feel like most muscle spasms feel. Light-pressure skin-surface palpation helps make fine adjustments in point location, while large pinches of the soft tissues around the knee are best for locating entire areas of abnormalities and often lead to unexpected deviations in traditional point locations.

\section{CONCLUSIONS}

From the author's personal experience, acupuncture appears to yield better results if acupuncture practitioners rely on the Four Examinations before considering the use of more-advanced methods. Careful visual and-even more importantly - palpatory evaluation of the patients knee and areas adjacent to the knee leg often reveals that the cause of the pain is not arthritis. Instead, the cause is nerve entrapment in soft-tissue adhesions and fibrosis-which are wellknown for causing muscle pain but rarely addressed in detail in acupuncture treatments. While the success of an acupuncture treatment can be measured after the knee pain is gone, locating and then needling those points where 
pressure reproduced the patients' pain appear to be essential in increasing the success rate of acupuncture treatments for knee pain as well as allowing practitioners to monitor the progress of their treatments.

\section{AUTHOR DISCLOSURE STATEMENT}

No competing financial interests exist.

\section{FUNDING INFORMATION}

This research did not receive any specific grants from funding agencies in the public, commercial, or not-for-profit sectors.

\section{REFERENCES}

1. Zhang Q, Yue J, Sun Z, Lu Y. Acupuncture for chronic knee pain: A protocol for an updated systematic review. $B M J$ Open. 2016;6(2):e008027.

2. Petrofsky JS, Laymon MS, Alshammari FS, Lee H. Use of low level of continuous heat as an adjunct to physical therapy improves knee pain recovery and the compliance for home exercise in patients with chronic knee pain: A randomized controlled trial. J Strength Cond Res. 2016;30(11):31073115 .

3. Bennell KL, Ahamed Y, Jull G, et al. Physical therapistdelivered pain coping skills training and exercise for knee osteoarthritis: Randomized controlled trial. Arthritis Care Res. 2016;68(5):590-602.

4. MacPherson H, Vickers A, Bland M, et al. Comparison of acupuncture with other physical treatments for pain caused by osteoarthritis of the knee: A network meta-analysis. In: Acupuncture for Chronic Pain and Depression in Primary
Care: A Programme of Research. Southampton, UK: NIHR Journals Library; 2017.

5. Fan AY, Zhou K, Gu S, Li YM. Acupuncture is effective for chronic knee pain: A reanalysis of the Australian acupuncture trial. Altern Ther Health Med. 2016;22(3):32-36.

6. Siqueira APR, Beraldo LM, Krueger E, Ulbricht L. Reduction in knee pain symptoms in athletes using an acupuncture protocol. Acta Ortop Bras. 2018;26(6):418-422.

7. Helianthi DR, Simadibrata C, Srilestari A, Wahyudi ER, Hidayat R. Pain reduction after laser acupuncture treatment in geriatric patients with knee osteoarthritis: A randomized controlled trial. Acta Med Indones. 2016;48(2):114-121.

8. Hinman RS, McCrory P, Pirotta M, et al. Acupuncture for chronic knee pain: A randomized clinical trial. JAMA. 2014; 312(13):1313-1322.

9. Zhang W, Doherty MA, Peat G, et al. EULAR evidencebased recommendations for the diagnosis of knee osteoarthritis. Ann Rheum Dis. 2010;69(3):483-489.

10. Li ZB, Kollias HD, Wagner KR. Myostatin directly regulates skeletal muscle fibrosis. J Biol Chem. 2008;283(28):19371-19378.

11. Yu XC, Gao JH, Fu WX. Ashi-points and the specificity of acupoints. Acupunct Res (Chin). 2005;30(3):183-190.

12. Shudo D; Brown S. (transl). Acupuncture Points and Palpation. Finding Effective Acupuncture Points, 1st ed. Seattle: Eastland Press; 2003:8:1-13.

13. Kong J, Gollub R, Huang T, et al. Acupuncture De Qi, from qualitative history to quantitative measurement. J Altern Complement Med. 2007;13(10):1059-1070.

14. Korostyshevskiy V. Locating active acupuncture points: The solution to a paradox in acupuncture research? J Chin Med. 2015;108:32-38.

Address correspondence to: Vladislav Korostyshevskiy, LAc, MS, MEd 2678 Ocean Avenue, Apartment $2 E$ Brooklyn, NY 11229 USA E-mail:vk546@nyu.edu 